\title{
A framework of genetic algorithm-based CNN on multi-access edge computing for automated detection of COVID-19
}

\author{
Md Rafiul Hassan ${ }^{1}$ - Walaa N. Ismail ${ }^{2}$. Ahmad Chowdhury ${ }^{3}$. Sharara Hossain ${ }^{4}$. \\ Shamsul Huda ${ }^{5}$ Mohammad Mehedi Hassan ${ }^{6}$ (D)
}

Accepted: 17 November 2021 / Published online: 21 January 2022

(c) The Author(s), under exclusive licence to Springer Science+Business Media, LLC, part of Springer Nature 2021, corrected publication 2023

\begin{abstract}
This paper designs and develops a computational intelligence-based framework using convolutional neural network $(\mathrm{CNN})$ and genetic algorithm (GA) to detect COVID-19 cases. The framework utilizes a multi-access edge computing technology such that end-user can access available resources as well the CNN on the cloud. Early detection of COVID-19 can improve treatment and mitigate transmission. During peaks of infection, hospitals worldwide have suffered from heavy patient loads, bed shortages, inadequate testing kits and short-staffing problems. Due to the timeconsuming nature of the standard RT-PCR test, the lack of expert radiologists, and evaluation issues relating to poor quality images, patients with severe conditions are sometimes unable to receive timely treatment. It is thus recommended to incorporate computational intelligence methodologies, which provides highly accurate detection in a matter of minutes, alongside traditional testing as an emergency measure. CNN has achieved extraordinary performance in numerous computational intelligence tasks. However, finding a systematic, automatic and optimal set of hyperparameters for building an efficient CNN for complex tasks remains challenging. Moreover, due to advancement of technology, data are collected at sparse location and hence accumulation of data from such a diverse sparse location poses a challenge. In this article, we propose a framework of computational intelligence-based algorithm that utilize the recent $5 \mathrm{G}$ mobile technology of multi-access edge computing along with a new CNN-model for automatic COVID-19 detection using raw chest X-ray images. This algorithm suggests that anyone having a 5G device (e.g., 5G mobile phone) should be able to use the CNN-based automatic COVID-19 detection tool. As part of the proposed automated model, the model introduces a novel CNN structure with the genetic algorithm (GA) for hyperparameter tuning. One such combination of GA and $\mathrm{CNN}$ is new in the application of COVID-19 detection/classification. The experimental results show that the developed framework could classify COVID-19
\end{abstract}

Extended author information available on the last page of the article 
X-ray images with $98.48 \%$ accuracy which is higher than any of the performances achieved by other studies.

Keywords Classification · CNN · COVID-19 · Genetic Algorithm · Multi-access edge

\section{Introduction}

An automatic detection of Coronavirus disease 2019 (COVID-19) can be developed through using the modern computational intelligence techniques and resources available on the high-performance computing facilities, e.g., cloud. The advent of convolutional neural network $(\mathrm{CNN})$, a variant computational intelligence $(\mathrm{CI})$ technique, has made the task of feature extraction from images and image analysis efficient. Moreover, the availability of high-performance computing (HPC) facilities, e.g., distributed edges on cloud, can help us to access COVID-19 data scattered at distant locations Coronavirus disease 2019 (COVID-19) is a highly contagious viral disease. It is caused by severe acute respiratory syndrome coronavirus 2 (SARSCoV-2). The first case of COVID-19 was identified in Wuhan, China, in December 2019, which eventually led to the ongoing pandemic [13, 33]. The World Health Organization declared the outbreak a Public Health Emergency of International Concern (PHEIC) in January 2020 and a pandemic in March 2020. COVID-19 symptoms include cough, fever or chills, shortness of breath or difficulty breathing, muscle or body aches, sore throat, temporary loss of taste or smell, diarrhea, headache, new fatigue, nausea or vomiting, and congestion or runny nose [25]. In more severe cases, the infection can cause pneumonia, the Middle East respiratory syndrome coronavirus (MERS-CoV), severe acute respiratory syndrome, kidney failure, and even death [19]. Diagnosis and prognosis of COVID-19 usually takes hours. Design and development of an automated system for rapid diagnosis/prognosis of COVID-19 will help to control spread of such epidemic. In this paper, we propose an automated model that uses genetic algorithm (GA) in developing a novel and effective CNN referred to as CGFor-Covid, which can assist experts in diagnosing COVID-19 from chest X-ray images through utilizing the state-of-art multi-access edge-computing $5 \mathrm{G}$ access network.

It is estimated that within the first ten months of the COVID-19 pandemic, suspected cases surpassed $10 \%$ of the global population (WHO/AP). As of December 25, 2020, 78,194,947 confirmed cases of COVID-19, including 1,736,752 deaths were reported to the WHO from around the world [8]. Traditionally, COVID-19 is diagnosed through Reverse Transcriptase Polymerase Chain (RT-PCR) testing, which has a sensitivity of $37.71 \%$ [9]. This test is expensive and takes several hours to diagnose the outcome $[7,8]$. Chest radiography and computed tomography (CT) are also key tools in the diagnosis of lung diseases [22]. Using X-ray radiography is cheap, readily available, and the devices can be cleaned easily.

In this study, we propose a framework that utilizes the recent $5 \mathrm{G}$ technology that utilizes the multi-access edge computing facilities because, these technologies help 


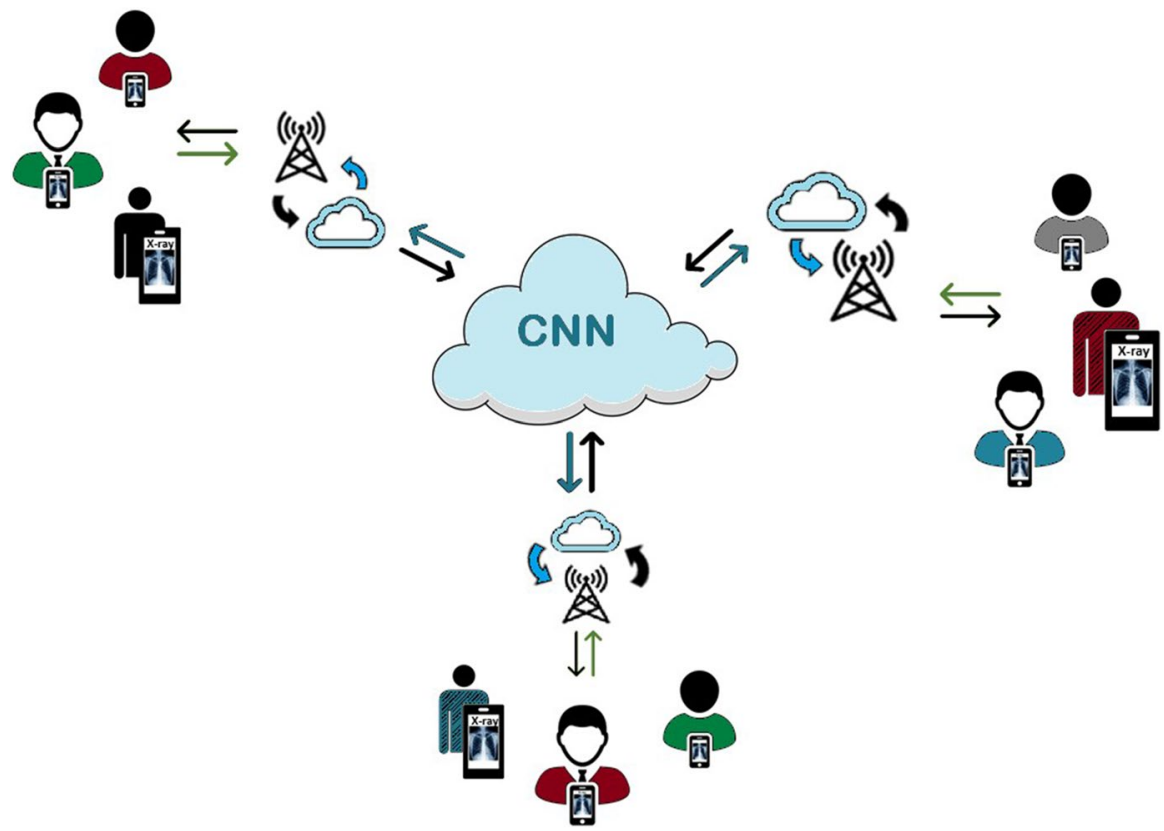

Fig. 1 Proposed framework for automated detection of COVID-19 through using multi-access edge computing and $\mathrm{CNN}$

the end-user to use available resources on the cloud with convenience. Figure 1 shows the proposed framework. As shown in the framework, data are accumulated at the central cloud from sparse location through a number of $5 \mathrm{G}$ edge and access network. Note that, the edge framework is used once the $\mathrm{CNN}$ is trained and optimized using a large training dataset. The trained CNN is thus stored in the central cloud from where every end-user can access the CNN through 5G edges. The 5G access network would help in accessing massive data volume with high throughput while the $5 \mathrm{G}$ edge would help in executing the low-latency applications, in this framework, the CNN. The idea is to allow access of the CNN by end-user at hand held $5 \mathrm{G}$ devices (e.g., smart phone or tablet/iPAD). If end-user can provide the COVID19 X-ray image, the CNN in the central cloud will be used to classify the image to either COVID-19 positive or negative case. The success of the proposed framework not only depends on the low-latency high-bandwidth multiple-access edge computing but also the classification accuracy of the CNN. Hence, we also design and develop an automated computational tool based on Artificial Intelligence (AI) techniques to diagnose COVID-19 using X-rays accurately. The subsequent sections of this article puts focus toward developing one such new AI techniques so that the developed technique can be used in the proposed framework as shown in Fig. 1.

In recent years, the adoption of deep learning (DL), a newly developed AI method, has about revolutionary changes in AI research and applications. Convolutional neural network (CNN), a variant of DL, is a widely used deep learning framework in biomedical image classification. A CNN algorithm can be used to extract 
features from blocks of convolutional and backpropagation approaches, which in turn utilizes one or more pooling layers or fully connected layers. The extracted features are then mapped into classification using a classifier. The performance of the CNN depends on the initial parameters (e.g., the structure of the CNN, the size of the convolution kernel matrix, which is known as the kernel, and so on). These initial parameters are pre-selected by the user for a given problem. Many studies have used CNN for COVID-19 detection by analyzing chest X-ray images. For example, Tulin Ozturk et al. [20] proposed a fully automated DarkCovidNet model (a CNN model) for COVID-19 classification and achieved an accuracy of 98.08\%. However, it is unclear how the authors chose the CNN structure. One of the challenges associated with the use of $\mathrm{CNN}$ is to identify a suitable structure for a given problem so that the best classification performance can be achieved. In our study, we propose and develop a deep CNN model in which a genetic algorithm (GA) is used to identify a suitable structure for efficiently classifying X-ray images for COVID-19 detection. The approach of combining GA with neural network architecture was inspired by previous studies that have investigated hyperparameter optimization for neural networks $[5,14,17]$. GA has been used to solve optimization problems in various fields, including autonomous crack detection [4], electromagnetics [1], synthesis of antenna patterns [16], computer vision and speech processing [36]. To the best of author's knowledge, the technique has not yet been applied to COVID-19 detection. Therefore, the proposed model uses this technique for the first time to classify X-ray images for COVID-19 detection.

In summary, the contributions of the current study are as follows:

1. Proposing a framework through utilizing the state-of-art multi-access edge computing, 5G access network and automated CNN to allow end-users access to the CNN for automatic diagnosis of chest X-ray images.

2. Developing a novel and effective CNN model optimized using a GA for COVID (CGForCovid), which can assist experts in diagnosing COVID-19 from chest $\mathrm{X}$-ray images.

3. Using a GA algorithm to accelerate the $\mathrm{CNN}$ architecture design by selecting the optimal parameters for the CNN architectures, thereby enhancing model performance by reducing the search space and decreasing the computational complexity.

4. Identifying the specific layer of a multilayer $\mathrm{CNN}$ structure that provides features that enhance classification. The features extracted at each layer of the CNN are fed into the classifier individually and classification performance is analyzed.

5. Conducting extensive experiments and comparing the results with the classification performances of systems reported in other recent studies.

\section{Related work}

The world has experienced significant social and economic disruption since the beginning of the COVID-19 pandemic. These problems can be solved, or at least curbed through quick and reliable COVID-19 detection by deploying AI tools ([6, 
11, 34]). Many studies have attempted to detect COVID-19 cases automatically using computational techniques. Given that this study focuses on detecting COVID19 from X-ray images, this section reviews existing studies that used X-ray images for COVID-19 case identification.

A comparative study was undertaken by the authors in [8] of RT-PCR and chest CT during the peak of the Italian epidemic, showing high sensitivity and specificity scores for detection using chest CT images. Tulin Ozturk et al. [20] proposed a fully automated DarkCovidNet model with a binary classification accuracy of 98.08\%. In the first few months of the pandemic, medical imaging data were scarce, and so to tackle this issue, Abdul Waheed et al. [29] used generative adversarial network (GAN) to generate new data and CNN for detection. The authors' COVIDGAN model displayed an improved accuracy of 95\%, where training on the original data yielded $85 \%$ accuracy. COVID-NET, a deep CNN model using open access data, has also been proposed [30]. A framework consisting of seven deep learning architectures such as VGG19, ResNetV2, InceptionV3 and MobileNetV2, known as COVIDX-Net yielded an F1-score of up to 0.91 [12]. A rather novel algorithm referred to as Capsnet, which was designed for image classification, has been used in Convolutional Capsnet [28], which gives $97.24 \%$ accuracy on binary classification. A transfer learning-based model nCOVnet achieved $88 \%$ overall accuracy and 97.62\% true positive rate [21]. Existing $\mathrm{CNN}$ architecture design algorithms require extensive work to manually design the $\mathrm{CNN}$ architecture, which often show slow performance for the complete model design. However, for users who may lack domain knowledge on CNNs, it is necessary to have powerful design that can offer an automatic way to tune model structure. GAs are inspired by the way biological development works, which involves selecting the population, and outputting more than one solution without being stuck to a local optimum [23, 32]. Therefore, GA can perform efficient optimization operations for selecting the optimal hyperparameter for deep neural network-based models. The researchers also implemented different optimization models for the selection of CNN hyperparameters using GA.

In NSANet [27], a new genetic CNN method was discussed for encoding the $\mathrm{CNN}$ architecture in multiple phases to replace the convolutional layers to build the final CNN. For each phase, the model architecture is gradually developed as a small unit known as a "cell" in Genetic CNN, and afterward, multiple building blocks of CNNs are ordered and encoded. The order of such building blocks is manually adjusted based on the first and last building blocks, and a binary string encoding method is used to encode the blocks connections. Setting all of these parameters is figured out manually with no techniques for acceleration having been designed, and this limits the applicability of the proposed model for complex datasets such as $\mathrm{X}$-ray images. A population-based training (PBT) algorithm for utilizing GA for neural network model hyperparameter selection was presented in [14]. PBT serves as a practical way to augment the standard training of neural network models using adaptive schedules. The proposed algorithm led to an outstanding improvement in the performance of neural network-based models, including hierarchical reinforcement learning, deep reinforcement learning, GANs, and machine translation. In [37], the authors proposed two hybrid models for the prediction and classification of HGBanemia, nutritional anemia, deficiency anemia, and folate deficiency anemia. The 
model worked by initiating the GA for the purpose of selecting the optimal hyperparameters for the stacked autoencoder (SAE) and CNN. The system achieved a classification accuracy of $98.50 \%$. In [27], GA was used to automate the CNN architecture design method. The authors targeted their work toward inexperienced users who lack CNN domain knowledge, specifically due to the value of their algorithm in automatically selecting the optimal CNN architecture to address the image classification process with $96.78 \%$ accuracy. Bakhshi et al. [3] proposed fast-CNN, a rapid and automatic $\mathrm{CNN}$ building architecture for image classification. Fast-CNN uses GA to define suitable CNN optimization parameters, including the learning rate, number of layers, momentum, number of feature maps, and weight decay factor. Similarly, in [35], the authors sought to solve the same problem using a variable length GA. The algorithm automatically searches for a CNN's optimal hyperparameters considering the expected depth increases of the CNN model and search space, which grows the number of hyperparameters selection time.

Based on these recent studies, and also inspired by the successful optimization of CNN hyperparameters using GA, this study proposes the application of a GA-initiated automatic CNN architecture design for COVID-19 X-ray image classification.

\section{Overview of the method}

In this study, we propose and develop the so-called CGForCovid model, a CNN structure for COVID-19 classification that uses GA to optimize the hyperparameters and determine suitable kernel sizes for the CNN structure. A kernel is a matrix that acts like a filter and moves over the input data, performing the dot product with the sub-region of input image. Figure 2 shows a schematic diagram of the proposed CGForCovid model. As shown in the figure, the model receives the COVID-19 $\mathrm{X}$-ray image dataset as input, and after preprocessing the dataset, two datasets (training/test sets) are prepared for the experiment evaluation. Afterward, the training

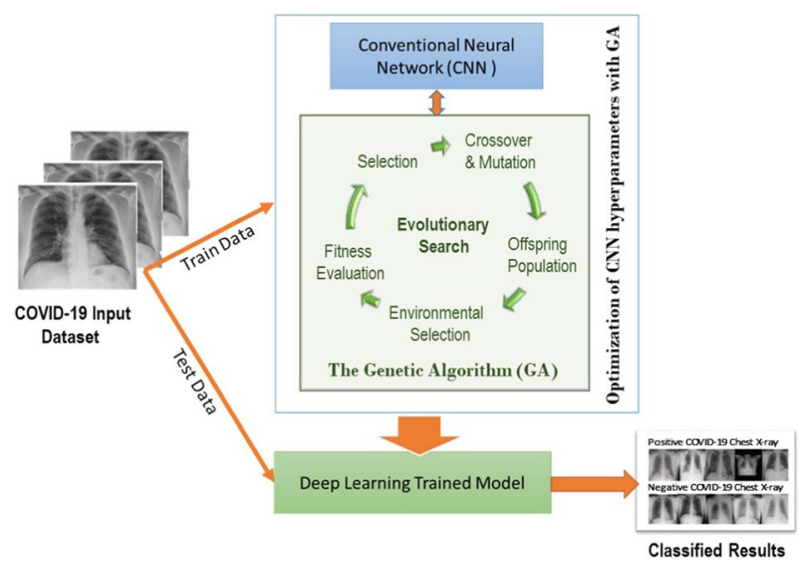

Fig. 2 The proposed CGForCovid model 
dataset is used as input to the CNN algorithm. The GA is used to select the hyperparameter values, thereby optimizing the $\mathrm{CNN}$ model. In the optimization process, GA starts with a range of values for kernel sizes as initial population, crossover and mutation, and the offspring population. GA is used to select the best and most significant combinations of the CNN model hyperparameters (i.e., kernel sizes). Finally, the achieved CNN model is used to classify patient health status. Figure 3 shows one such CNN architecture that can be used for COVID-19 X-ray images (RGB images of size $224 \times 224 \times 3$ ) classification. The following subsections describe how GA is used to optimize an initially chosen CNN structure.

Before describing the combined GA-CNN model, let us first introduce the GA and $\mathrm{CNN}$ in brief.

\subsection{The genetic algorithm}

GA is a variant of the popular evolutionary algorithm (EA). The difference between GA and EA is that in the latter, the chromosomes represent real numbers, while in GA chromosomes represent binary numbers. EA is the most feasible solution that is widely used for many optimization problems. The concept of EA is inspired by Darwinism, a biological evolutionary theory of nature that posits the survival of the fittest. This makes it significantly different compared to other search engines as it allows sampling through large search effectively. In EA/GA, an initial population is randomly generated as candidate solutions followed by three operations: selection, crossover, and mutation. Each individual solution is represented as a chromosome that consists of a set of strings, each with their own fitness value. Fitness value is a score (e.g., classification accuracy of a model) that determines how satisfactory the solution is. A population is the set of current sets of solutions (chromosomes) from which new sets of solutions are to be generated. To select the parent string from the current population, the probability of each string in the population of the

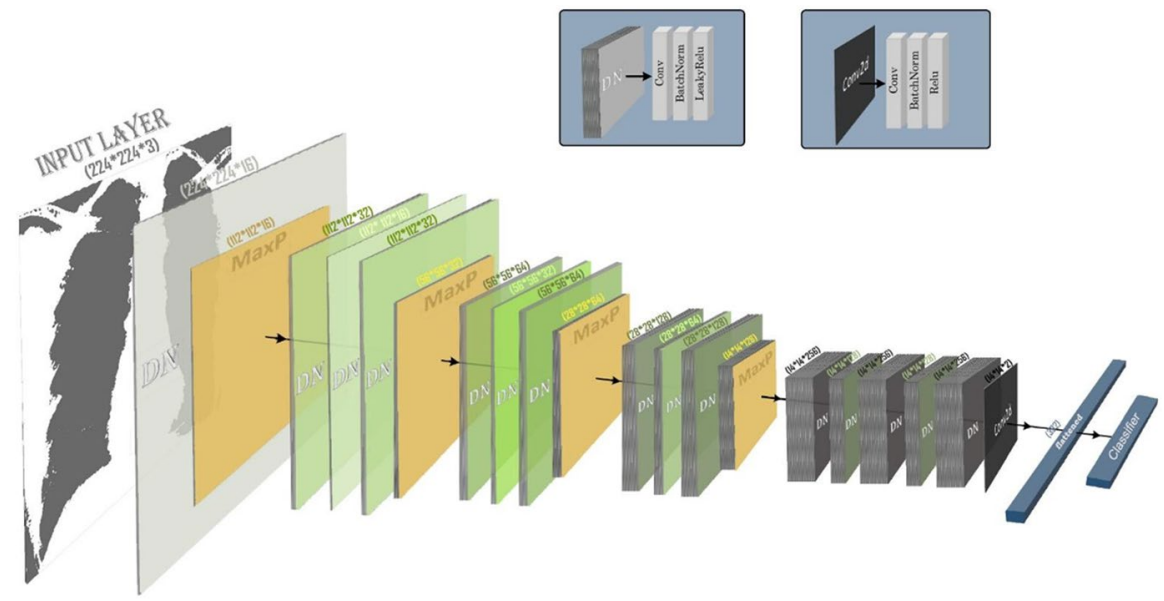

Fig. 3 An architecture of the CNN used in CGForCovid Model 
current generation is determined considering the fitness value. Crossover in GA generates new generations by mating parent solutions (chromosomes) by crossing over the strings at some point to produce offspring. Mutation introduces variation into the population with a time interval by changing certain bit values in the string (chromosome). These operations are executed iteratively to create new generations until a stopping criterion is reached. The objective of EA/GA is either to minimize or maximize the fitness value based on survival of the fittest criterion.

\subsection{Convolutional neural network}

A typical CNN structure has one or more convolutional layer (CNN kernel) that extracts the significant feature set from the input dataset with its own filters. There is one or more pooling layer used in the CNN. The pooling layer decreases the size of the intermediate features without losing significant information from the feature set. The last layer of the CNN is typically used to classify data using the extracted features. By consolidating the layers, a CNN model is constructed, and using a training algorithm and dataset, the internal encoding parameters of the CNN are adjusted to classify/predict COVID-19 cases. There is no known approach available for selecting the best CNN structure for a problem. While attempting to use CNN for COVID19 detection, we found that CNN kernel size affects classification performance. Therefore, optimization is important because poor classification accuracy may not necessarily be due to noisy data or algorithms suffering from weak learning; it also results from a poor combination of parametric values.

\subsection{Combining GA with CNN}

In our proposed CGForCovid model, chest X-ray images are used as input to the model. For each individual image input $\mathrm{X}$ and kernel $\mathrm{K}$, the following convolution operation is used,

$$
(X * K)(i, j)=\sum_{m} \sum_{n} K(m, n) X(i-m, j-n)
$$

where $*$ represents the discrete convolution operation. Here, the kernel matrix $\mathrm{K}$ is slid over the input matrix $\mathrm{L}(\operatorname{size}(L)>\operatorname{size}(M))$ to extract features through the convolution operation. After the convolution, the achieved features are further rectified using a nonlinear activation function. Leaky rectified linear unit (Leaky ReLu) has recently grown in popularity as an activation function because it does not change the size of the input and does not suffer from the vanishing gradient problem. In CGForCovid, Leaky ReLu is used as the rectifier at each convolutional layer. Equation 2 explains the calculation used in the Leaky ReLu function.

$$
\text { Leaky } \operatorname{ReLu}(x)= \begin{cases}0.01 x & \text { for } x<0 \\ x & \text { for } x \geq 0\end{cases}
$$


The GA used in CGForCovid has a single objective because only one fitness value/ score (i.e., classification accuracy for the training/validation dataset) is maximized using the standard GA operators of selection, crossover, and mutation. The chromosome of the GA is a potential solution/kernel size of the CNN for COVID-19 classification. As such the GA should provide a suitable kernel size for the CNN structure to maximize COVID-19 classification accuracy. Algorithm 1 shows the procedure used to identify suitable kernel size in pseudo-code.

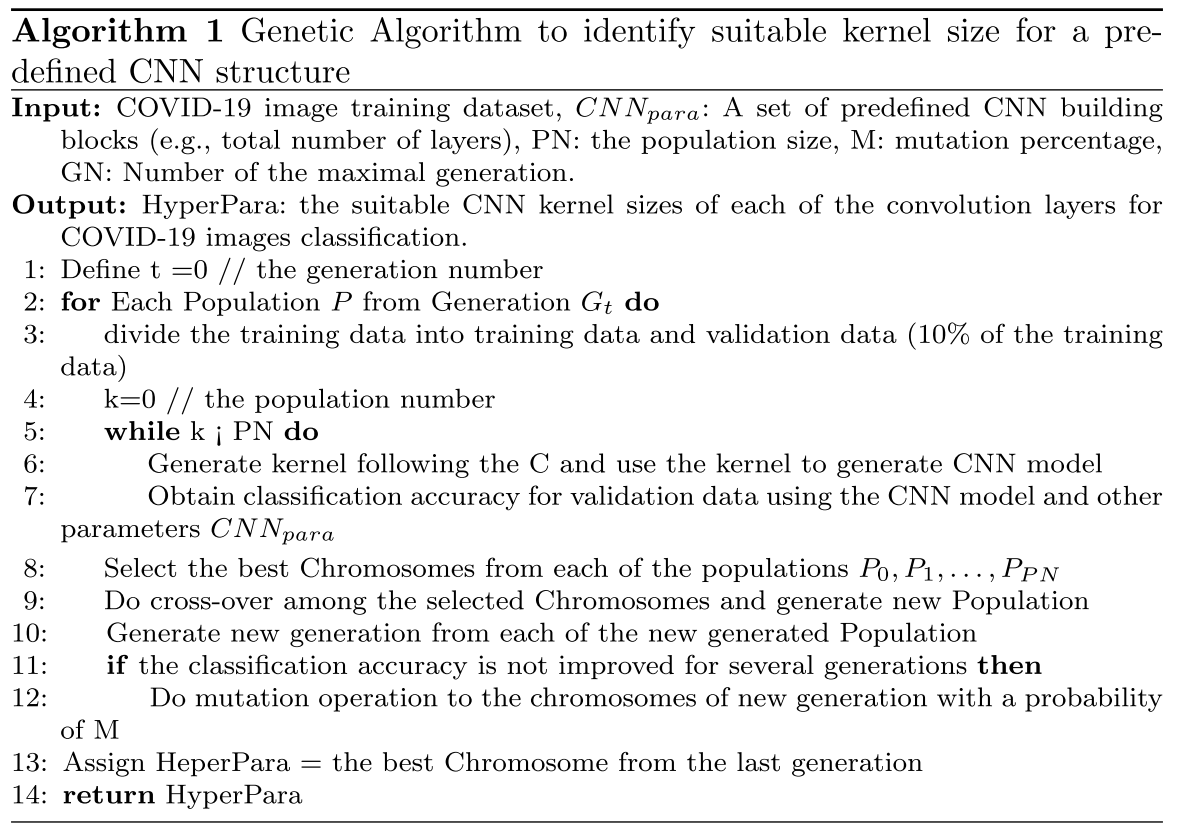

As described in the algorithm, it starts to build the CNN model with a defined population size and maximal generation number. The GA algorithm begins to work iteratively by searching for suitable optimization parameters (kernel size) for the CNN architecture to classify whether the patient is COVID-19-positive or not from the collected image dataset. To encode the $\mathrm{CNN}$ model, a set of iterations are conducted to randomly initialize a population with the predefined maximal population size $P N$; that is to say each generation consists of $P N$ populations. In the process of evolution, the fitness of each individual chromosome in each population is calculated. Once the best chromosomes are identified based on the fitness score, a new population is generated from the selected chromosomes through the crossover operation. This process of evolution continues up to a maximum generation, and the best chromosome in the last generation is regarded as the suitable kernel size for the CNN structure for COVID-19 detection. 


\section{Experimental setup and results}

\subsection{Experimental setup}

The proposed model utilizes GA for optimizing CNN structure. Following list of parameters for GA were used to conduct the experiment:

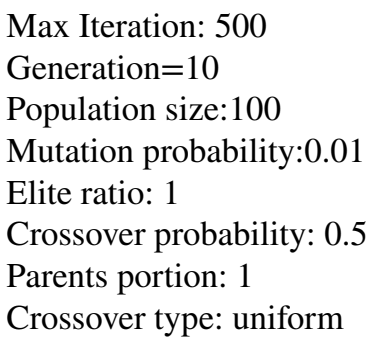

16-256 filters for each convolution layer were used in the proposed CGForCovid model. Each convolutional layer in the CNN was followed with a softmax layer, which is a fully connected dense neural network classifier, to observe accuracy performance before building up the next block of convolutional layers. To downsize the input X-ray images, the maxpool method was used in all the pooling operations. The maxpool method works by taking the maximum pixel value determined by its filter. The final CNN architecture was evaluated using fivefold cross-validation for different classifiers (several classifiers were used at the last layer of the $\mathrm{CNN}$ ), including Dense (traditional fully connected neural network), random forest, and decision tree. The model thus classifies the input X-ray images either as COVID-19 positive or COVID-19 negative cases.

To identify the layer of the CNN that provided the optimal features, a classifier was used at the end of each layer to classify the data using extracted features by the respective $\mathrm{CNN}$ layer. To determine which classifier in combination with the extracted features provided better classification performance, three different classifiers were tested: fully connected neural network classifier with the random forest classifier and decision tree. The performance of each stage/layer of the CNN architecture was investigated by training the models with categorical cross-entropy as the loss function, a learning rate of 0.0001 for the Adam optimizer, and using a fully connected neural network as a classifying layer.

To overcome the overfitting problem, $20 \%$ of the training X-ray data were used for validation and $80 \%$ for training the CNN model. Additionally, to test robustness of the CGForCovid model, fivefold cross-validation was used. The parameters of the models used in the study are shown in Tables 2 and 3. The Keras platform written in Python was used to conduct all experiments. In the study, all implementations were executed on a system with a 64-bit Intel Core I5 processor, and 12 GB of RAM. 


\subsection{Dataset}

X-ray images were obtained from a dataset developed by Joseph Paul Cohen and Paul Morrison and Lan Dao [15]. This dataset contains chest X-ray and CT scan images of COVID-19, MERS, SARS, and ARDS. The dataset includes frontal and lateral view imagery and metadata, including the time since first symptoms, survival status, and location. We opted to use X-ray images for our study, of which there are 420 COVID-19 positive cases and 505 COVID-19 negative cases in our X-ray dataset. Figure 4 shows example X-ray images of COVID-19 positive cases, and Fig. 5 illustrates X-ray images of COVID-19 negative cases from the dataset.

\subsection{Performance metrics}

For all the simulations, a binary classifier is initialized to predicts all the data instances with four possible outcomes: TP (true positive), TN (true negative), FP (false positive), and FN (false negative).

Fig. 4 COVID-19-positive $\mathrm{X}$-ray images
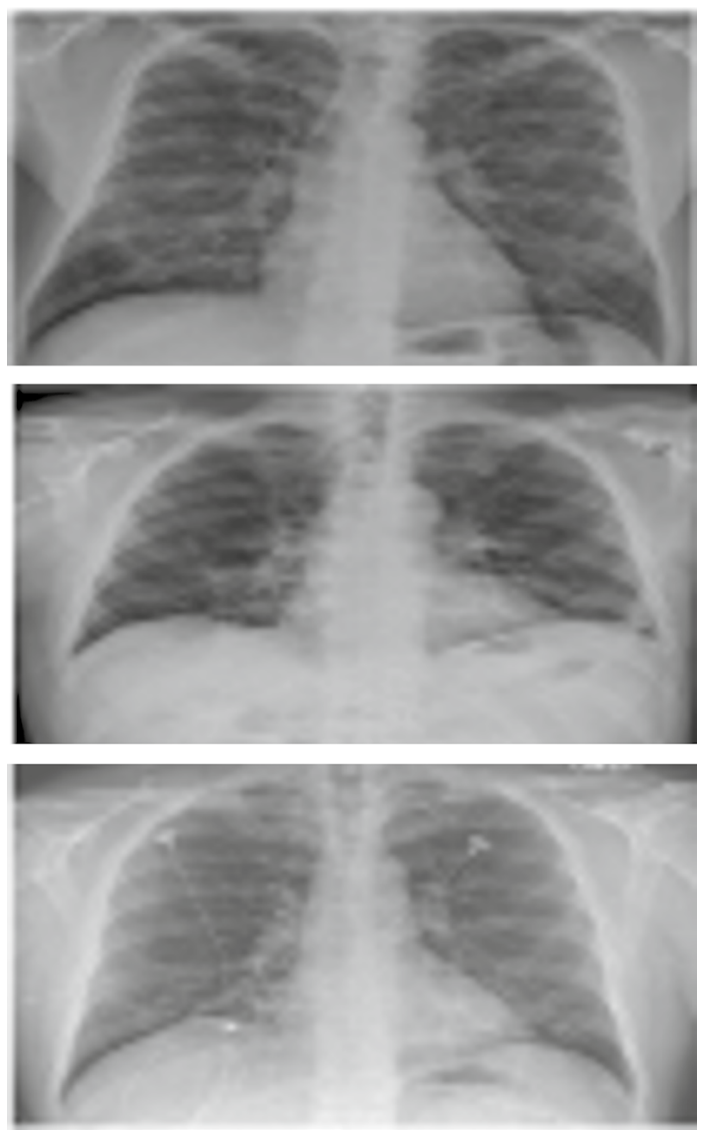

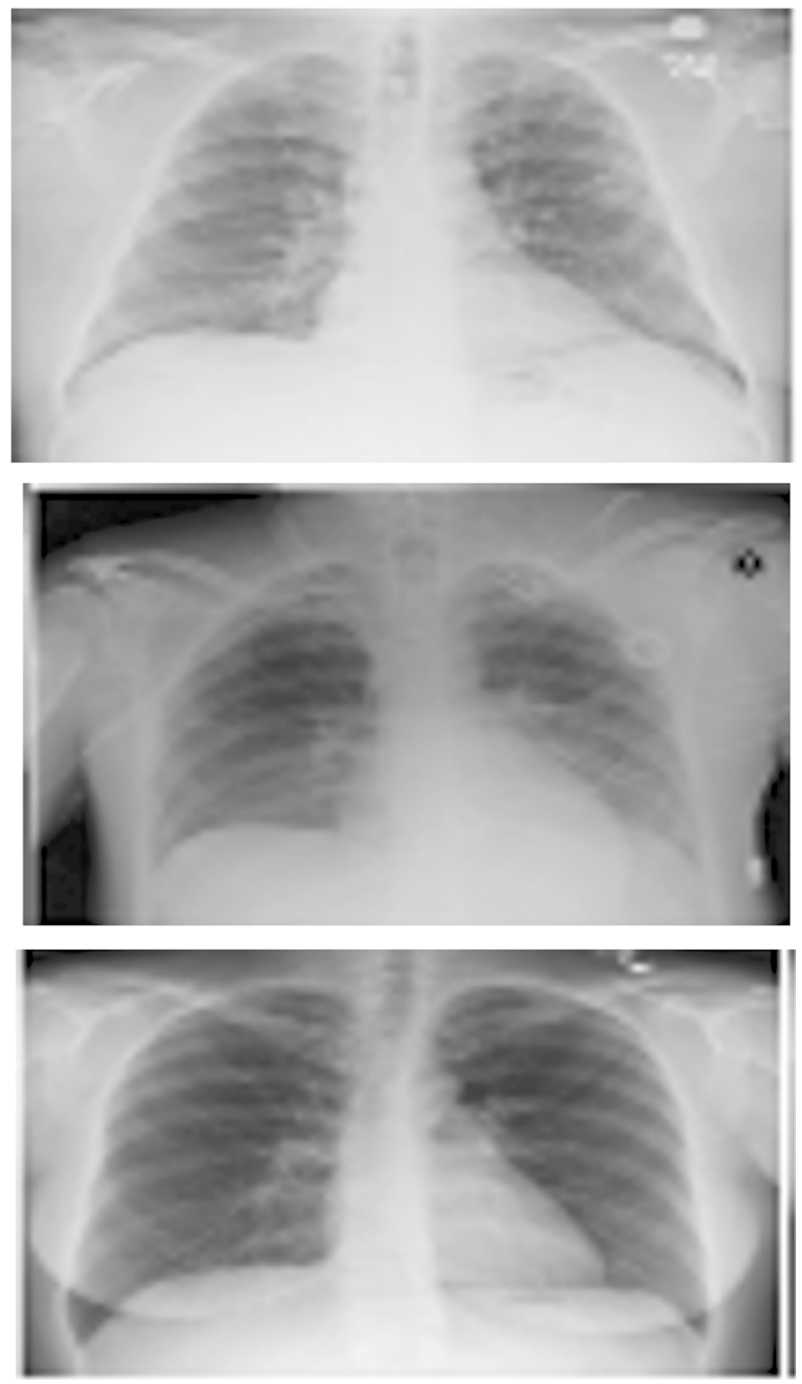

Fig. 5 COVID-19-negative X-ray images

- TP: correct positive prediction

- FP: incorrect positive prediction

- TN: correct negative prediction

- FN: incorrect negative prediction

Accuracy is calculated as the total number of two correct predictions $(\mathrm{TP}+\mathrm{TN})$ divided by the total number of a dataset $(\mathrm{P}+\mathrm{N})$.

- Accuracy $=(t p+t n) /(t p+t n+f p+f n)$ 
Sensitivity (recall or true positive rate) is calculated as the number of correct positive predictions (TP) divided by the total number of positives $(\mathrm{P})$.

- Sensitivity (recall or true positive rate $)=\mathrm{tp} /(\mathrm{tp}+\mathrm{fn})$

Specificity (true negative rate) is calculated as the number of correct negative predictions $(\mathrm{TN})$ divided by the total number of negatives $(\mathrm{N})$.

- Specificity $($ true negative rate $)=\mathrm{tn} /(\mathrm{tn}+\mathrm{fp})$

Precision (positive predictive value) is calculated as the number of correct positive predictions (TP) divided by the total number of positive predictions (TP $+\mathrm{FP})$.

- Precision $($ positive predictive value $)=\mathrm{tp} /(\mathrm{tp}+\mathrm{fp})$

F1 score is the harmonic mean of the precision and recall.

- $\mathrm{F} 1=(2 *$ precision*recall $) /($ precision + recall $)$

A receiver operating characteristic curve, or ROC curve, is a graphical plot that illustrates the diagnostic ability of a binary classifier system by plotting the true positive rate against the false positive rate at various threshold settings. When using normalized units, the area under the curve (AUC) of the ROC plot is equal to the probability that a classifier will rank a randomly chosen positive instance higher than a randomly chosen negative one [10].

\subsection{Experimental results of the proposed model}

In this study, a combination of state-of-art CI technologies: GA and CNN (CGForCovid) was proposed to classify the COVID-19 X-ray image dataset. Table 1 lists the detailed parameters achieved by using the proposed CGForCovid model for the selected dataset. The proposed model uses 16 convolution layers to classify COVID19 X-ray images. Compared to another CNN known as Darknet-19, that model used 19 convolutional layers to classify COVID-19 X-ray images. Moreover, the proposed model can be used for any COVID-19 dataset because the GA identifies a suitable CNN structure if the training dataset changes. In contrast, Darknet-19 is not adaptive under a change of training dataset.

Table 2 reports the experimental results for the dataset discussed in Section 2. To identify the layer of the CNN that can provide the best feature set for effectively identifying COIVD-19 cases, features extracted from each block (see column 1 in the table for block numbers) are individually fed into the respective classifiers to classify the data. As the table shows, block number 7 provided the best features that assisted in classifying the COVID-19 cases, achieving $98.91 \%$ accuracy. This experimental results demonstrate that the optimization of a CNN using GA can deliver COVID-19 classification with high accuracy, as well as a 98.907 AUC score. 
Table 1 The experimental hyperparameters of the CGForCovid model

\begin{tabular}{|c|c|c|c|}
\hline Layer \# & Block \# & Layer(type) & Output Shape Param \# \\
\hline 1 & 1 & conv $2 d$ & (None, 224, 224, 16) \\
\hline 2 & & batch_normalization & (None, 224, 224, 16) \\
\hline 3 & & LeakyReLU & (None, 224, 224, 16) \\
\hline 4 & & MaxPooling2D & (None, $112,112,16$ ) \\
\hline 5 & 2 & conv $2 d$ & (None, $112,112,32$ ) \\
\hline 6 & & batch_normalization & (None, $112,112,32$ ) \\
\hline 7 & & LeakyReLU & (None, $112,112,32$ ) \\
\hline 8 & & conv2d & (None, $112,112,16$ ) \\
\hline 9 & & batch_normalization & (None, $112,112,16$ ) \\
\hline 10 & & LeakyReLU & (None, $112,112,16$ ) \\
\hline 11 & & conv $2 d$ & (None, $112,112,32$ ) \\
\hline 12 & & batch_normalization & (None, $112,112,32$ ) \\
\hline 13 & & LeakyReLU & (None, $112,112,32$ ) \\
\hline 14 & & MaxPooling2D & (None, $56,56,32$ ) \\
\hline 15 & 3 & conv $2 d$ & (None, $56,56,64$ ) \\
\hline 16 & & batch_normalization & (None, 56, 56, 64) \\
\hline 17 & & LeakyReLU & (None, $56,56,64$ ) \\
\hline 18 & & conv2d & (None, 56, 56, 32) \\
\hline 19 & & batch_normalization & (None, $56,56,32$ ) \\
\hline 20 & & LeakyReLU & (None, $56,56,32$ ) \\
\hline 21 & & conv2d & (None, 56, 56, 64) \\
\hline 22 & & batch_normalization & (None, 56, 56, 64) \\
\hline 23 & & LeakyReLU & (None, 56, 56, 64) \\
\hline 24 & & MaxPooling2D & (None, 28, 28, 64) \\
\hline 25 & 4 & conv2d & (None, 28, 28, 128) \\
\hline 26 & & batch_normalization & (None, 28, 28, 128) \\
\hline 27 & & LeakyReLU & (None, 28, 28, 128) \\
\hline 28 & & conv2d & (None, 28, 28, 64) \\
\hline 29 & & batch_normalization & (None, 28, 28, 64) \\
\hline 30 & & LeakyReLU & (None, 28, 28, 64) \\
\hline 31 & & conv2d & (None, 28, 28, 128) \\
\hline 32 & & batch_normalization & (None, 28, 28, 128) \\
\hline 33 & & LeakyReLU & (None, 28, 28, 128) \\
\hline 34 & & MaxPooling2D & (None, $14,14,128$ ) \\
\hline 35 & 5 & conv2d & (None, $14,14,256$ ) \\
\hline 36 & & batch_normalization & (None, 14, 14, 256) \\
\hline 37 & & LeakyReLU & (None, $14,14,256$ ) \\
\hline 38 & & conv2d & (None, $14,14,128$ ) \\
\hline 39 & & batch_normalization & (None, $14,14,128$ ) \\
\hline 40 & & LeakyReLU & (None, $14,14,128$ ) \\
\hline 41 & & conv $2 d$ & (None, 14, 14, 256) \\
\hline 42 & & batch_normalization & (None, $14,14,256$ ) \\
\hline 43 & & LeakyReLU & (None, 14, 14, 256) \\
\hline
\end{tabular}


Table 1 (continued)

\begin{tabular}{llll}
\hline Layer \# & Block \# & Layer(type) & Output Shape Param \# \\
\hline 44 & 6 & conv2d & (None, 14, 14, 128) \\
45 & & $\begin{array}{l}\text { batch_normalization } \\
\text { LeakyReLU }\end{array}$ & (None, 14, 14, 128) \\
46 & & conv2d & (None, 14, 14, 14, 256) \\
47 & 7 & batch_normalization & (None, 14, 14, 256) \\
48 & & LeakyReLU & (None, 14, 14, 256) \\
49 & & conv2d & (None, 14, 14, 2) \\
50 & & batch_normalization & (None, 14, 14, 2) \\
51 & & flatten (Flatten) & (None, 392) \\
52 & dense (Dense) & (None, 2) \\
53 & &
\end{tabular}

Moreover, using different classifiers at the last level of the model, we sought to identify the optimally performing classifier. As the table shows, all three classifiers, namely neural network, decision tree, and random forest, achieved the same level of classification performance for COVID X-ray image classification. This demonstrates that the combined use of GA and $\mathrm{CNN}$ can generate features that are significant for COVID-19 classification.

After achieving an impressive classification performance for the training and test data $(80 \%$ of the data were randomly selected to train the model, while the remaining $20 \%$ were used to test the model), a fivefold cross-validation scheme was used to achieve a generalized performance of the proposed CGForCovid model. The graphs

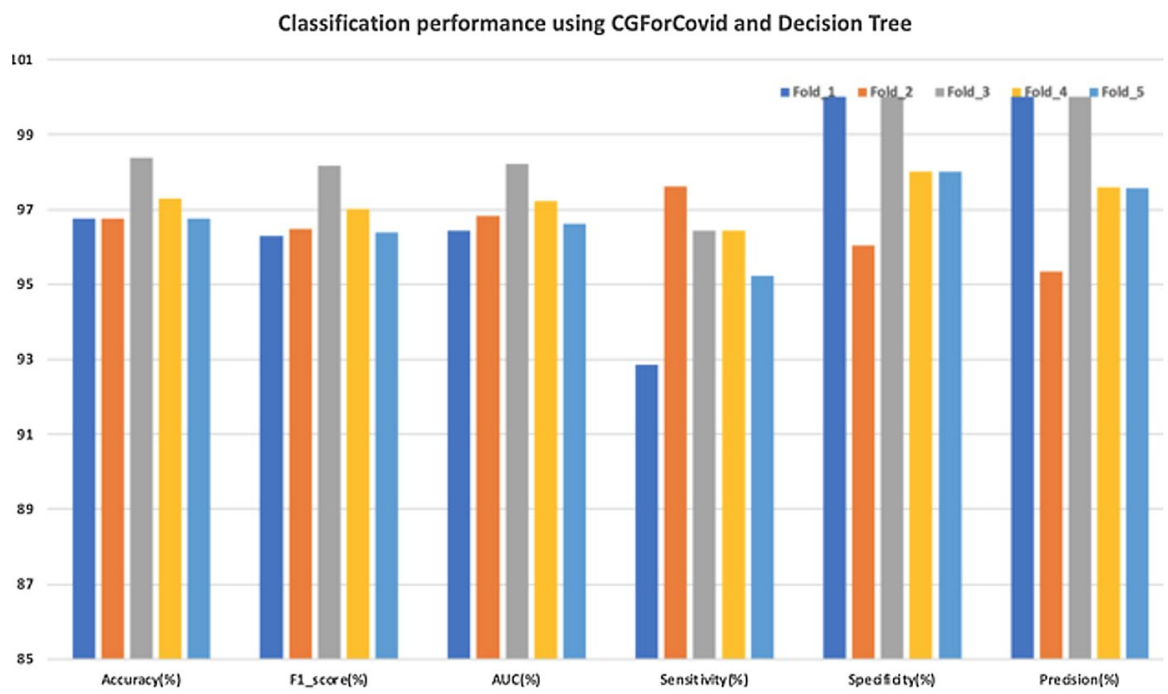

Fig. 6 Classification performances for each of the fivefolds using CGForCovid along with a decision tree at the last layer as classifier 


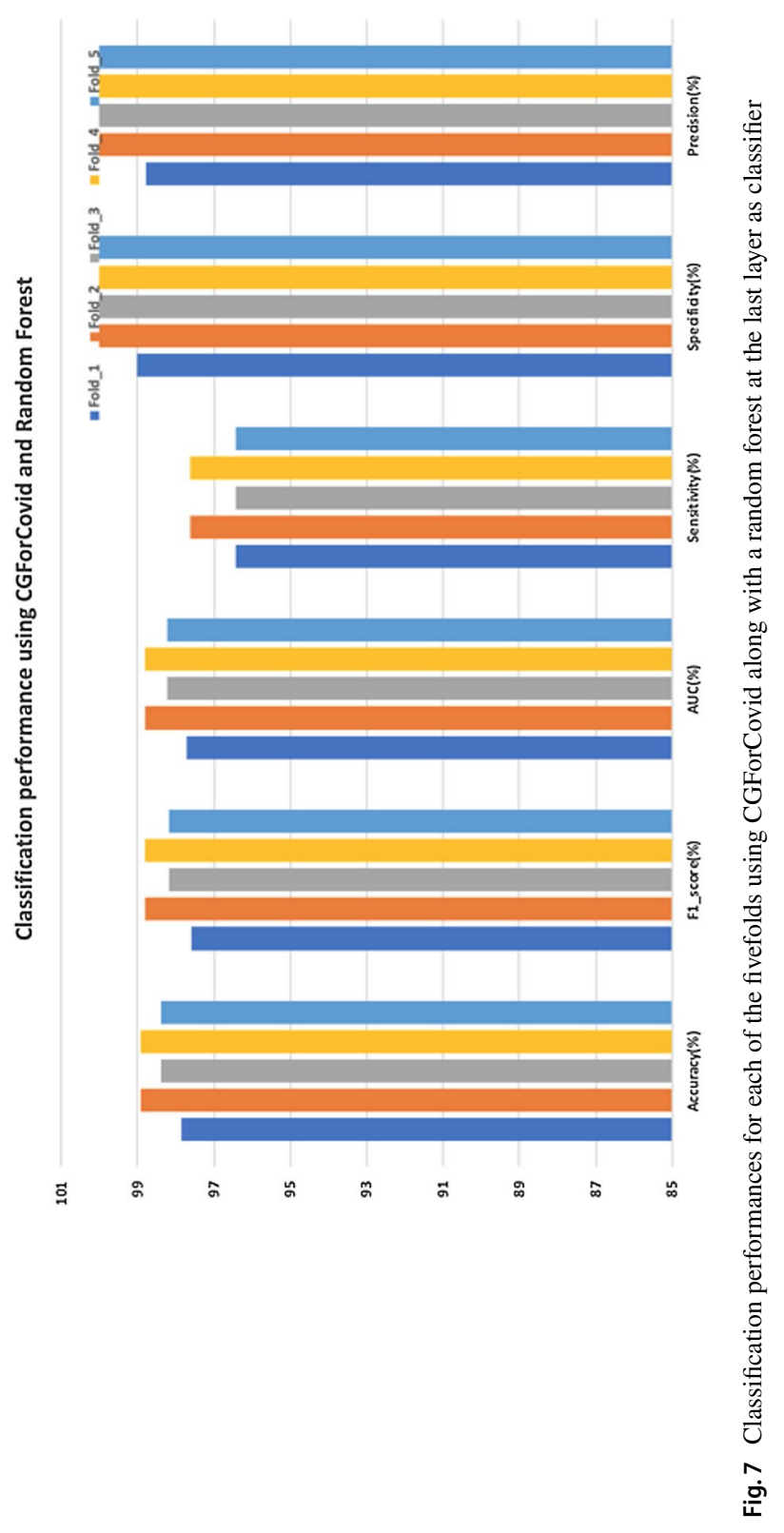




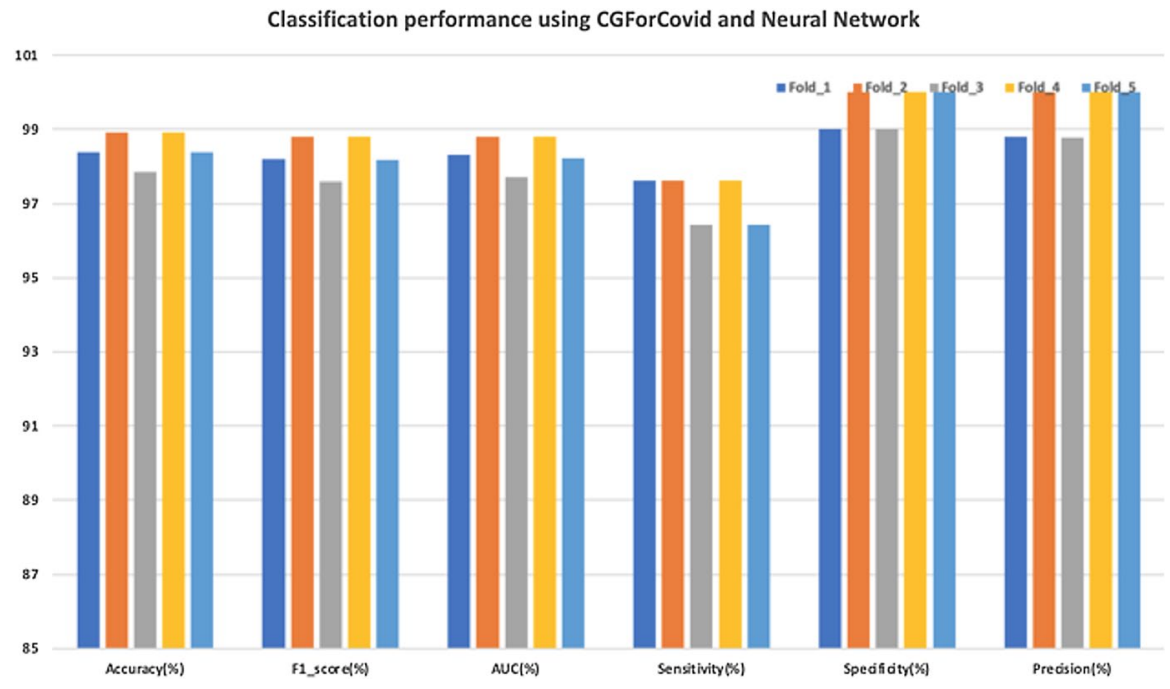

Fig. 8 Classification performances for each of the fivefolds using CGForCovid along with a neural network (i.e., softmax) at the last layer as classifier

in Figures 6, 7 and 8 show the performance variation in terms of different performance metrics (e.g., accuracy, F1-score, AUC, sensitivity, specificity, and precision) across different folds. Figures 6, 7, and 8 show that the performance variation is insignificant if the classifiers are varied that are used after having features extracted using the combination of GA and CNN. One such performance signifies that the GA-optimized CNN can extract influential features from the X-ray images. Table 3 shows the variation in classification performance among different classifiers (i.e., those that were used as the last layer of the $\mathrm{CNN}$ ) for fivefold cross-validation. As shown in the table, the classifier neural networks can achieve a better performance in terms of accuracy ( $98.49 \pm 0.45)$, F1-score $(98.31 \pm 0.50)$, AUC (98.37 \pm 0.46$)$, and Sensitivity $(97.14 \pm 0.65)$, while Random Forest can provide better Specificity $(99.80 \pm 0.44)$ and Precision scores $(99.76 \pm 0.55)$. Although neural networks can improve performance, the performance differences among these classifiers are negligible (especially between neural network and random forest). Moreover, the small standard deviation (e.g., for neural network the standard deviation for accuracy is 0.45) across the fivefold cross-validation scores indicates that the proposed model not only provide a very impressive classification performance for COVID-19 identification but also is a steady system.

Figure 9 shows the normalized confusion matrices of the models. The row of the matrix represents the instances in the actual class, while the column represents the instances in a predicted class. As the confusion matrix shows, no non-COVID cases were classified as COVID cases and only 12 out of 408 patients were misclassified as non-COVID cases. Certainly, such high classification accuracy would encourage physicians/radiologists/professionals to use the proposed automated COVID classifier. Figure 10 shows the ROC curve for the fivefold cross-validation test scheme. As 
Fig. 9 Confusion Matrix for CGForCovid model
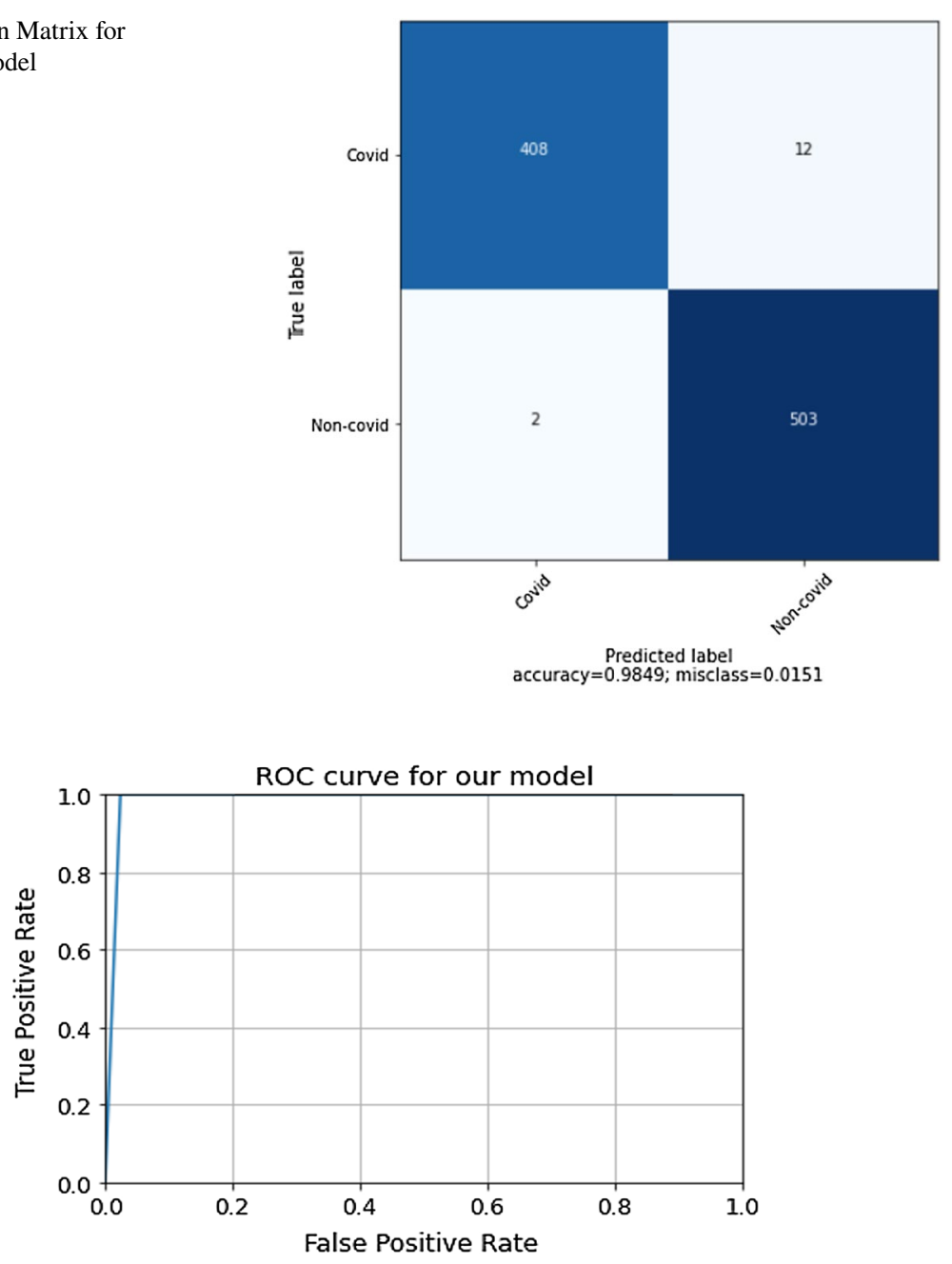

Fig. 10 CGForCovid model ROC Curve

usual, the curve is close to the point $(0,1.0)$, which reveals the effectiveness of the proposed classifier.

\section{Comparison with existing work}

Table 4 reports the COVID-19 classification performances achieved in several previous studies. As shown in the table, [2] used transfer learning with VGG-19 feature extraction model for input images, achieving an accuracy of $93.48 \%$. Our proposed model's performance is $5 \%$ better than that of the model of [2]. Similarly, our model is at least $6.08 \%$ better than that of Wang and Wong [30], 3.1\% better than that of 


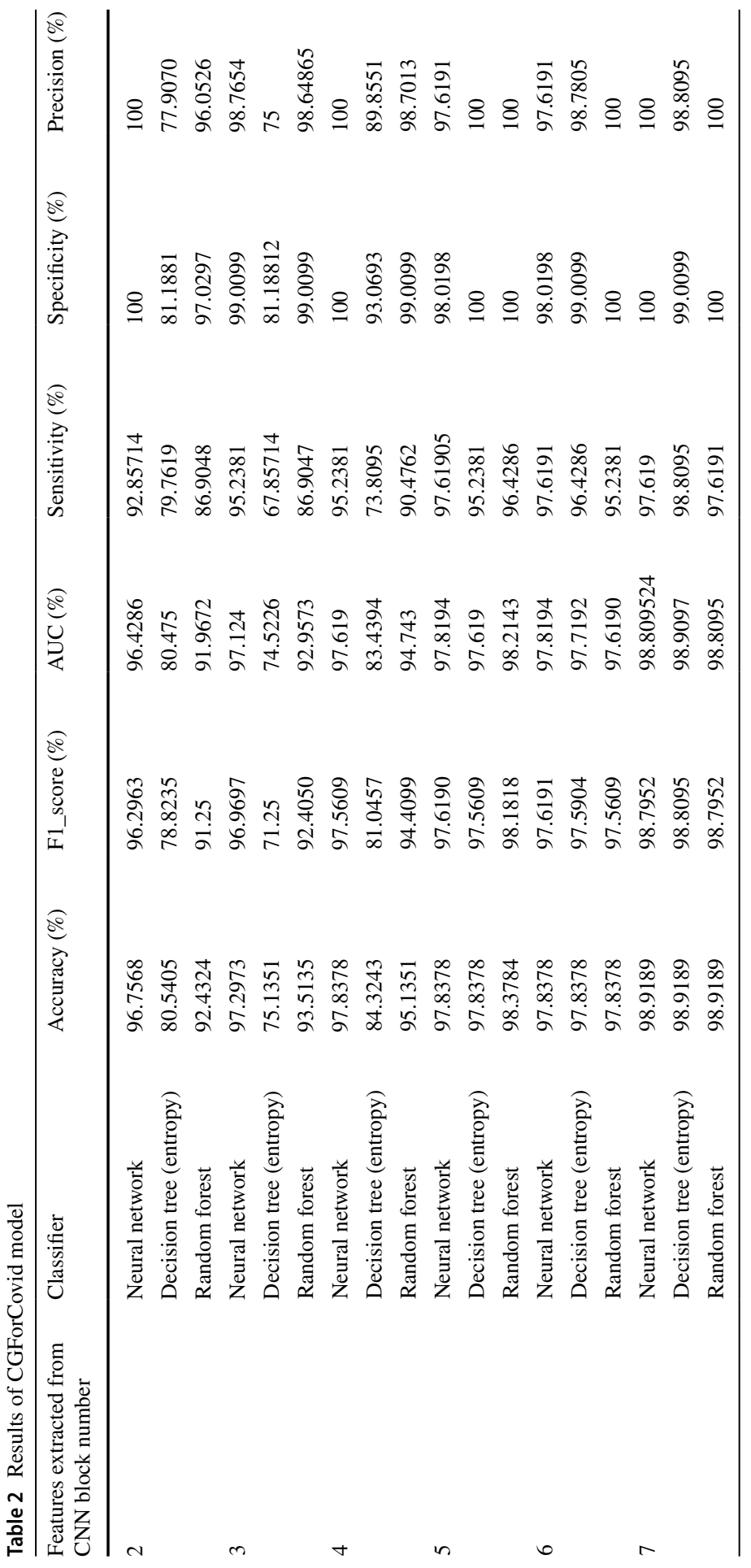




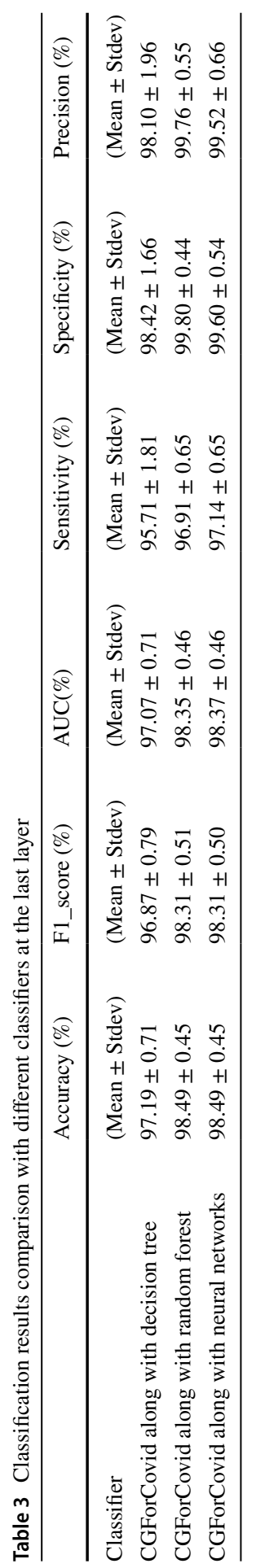




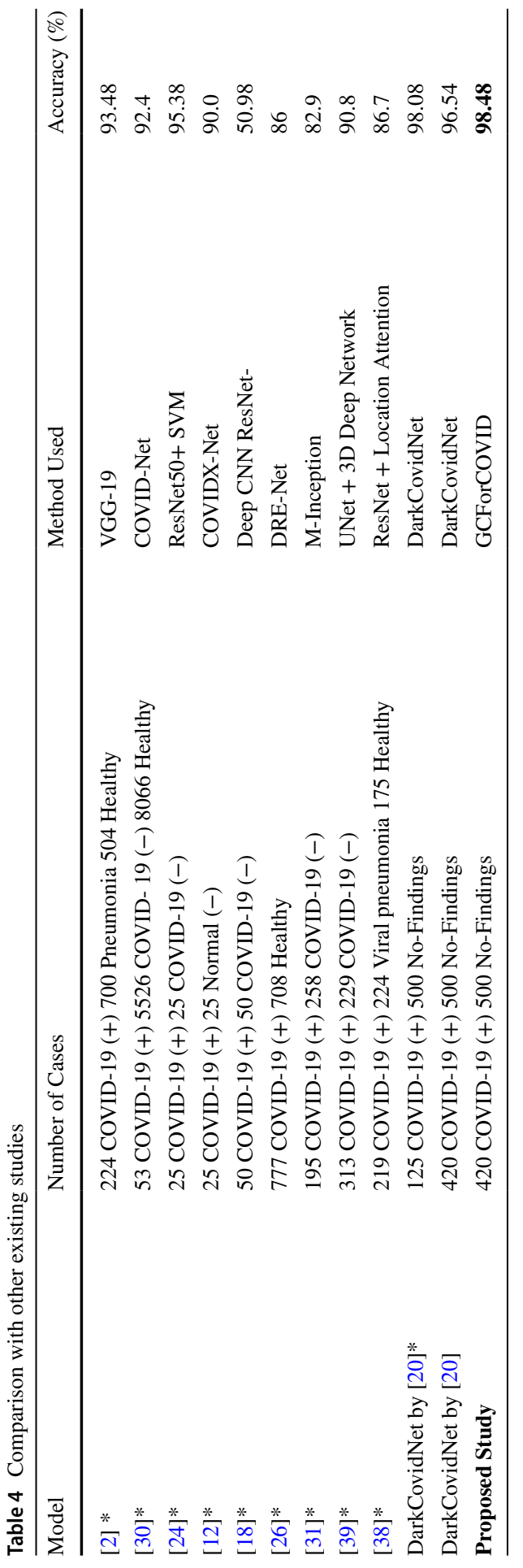


Table 5 List of parameters to design and develop DarkCovidNet-19 Model

\begin{tabular}{llll}
\hline Type & Filters & Size/Stride & Output \\
\hline Convolutional & 32 & $3 \times 3$ & $224 \times 224$ \\
Maxpool & & $2 \times 2 / 2$ & $112 \times 112$ \\
Convolutional & 64 & $3 \times 3$ & $112 \times 112$ \\
Maxpool & & $2 \times 2 / 2$ & $56 \times 56$ \\
Convolutional & 128 & $3 \times 3$ & $56 \times 56$ \\
Convolutional & 64 & $1 \times 1$ & $56 \times 56$ \\
Convolutional & 128 & $3 \times 3$ & $56 \times 56$ \\
Maxpool & & $2 \times 2 / 2$ & $28 \times 28$ \\
Convolutional & 256 & $3 \times 3$ & $28 \times 28$ \\
Convolutional & 128 & $1 \times 1$ & $28 \times 28$ \\
Convolutional & 256 & $3 \times 3$ & $28 \times 28$ \\
Maxpool & & $2 \times 2 / 2$ & $14 \times 14$ \\
Convolutional & 512 & $3 \times 3$ & $14 \times 14$ \\
Convolutional & 256 & $1 \times 1$ & $14 \times 14$ \\
Convolutional & 512 & $3 \times 3$ & $14 \times 14$ \\
Convolutional & 256 & $1 \times 1$ & $14 \times 14$ \\
Convolutional & 512 & $3 \times 3$ & $14 \times 14$ \\
Maxpool & & $2 \times 2 / 2$ & $7 \times 7$ \\
Convolutional & 1024 & $3 \times 3$ & $7 \times 7$ \\
Convolutional & 512 & $1 \times 1$ & $7 \times 7$ \\
Convolutional & 1024 & $3 \times 3$ & $7 \times 7$ \\
Convolutional & 512 & $1 \times 1$ & $7 \times 7$ \\
Convolutional & 1024 & $3 \times 3$ & $7 \times 7$ \\
Convolutional & 1000 & $1 \times 1$ & $7 \times 7$ \\
Avgpool & & & \\
Softmax & & & \\
\hline & & & \\
\hline
\end{tabular}

Sethy and Behra [24], and $8.48 \%$ better than that of Hemdan et al. [12]. As the table reports, outperformed all others that have recently been proposed in terms of classification performance. It should be noted that all the classification accuracies with ' $*$ ' symbols are reported by the respective studies. Hence, the comparison might not be fair. For a fair comparison, the same dataset and data distribution among the fivefolds should be used. In our experiment, we implemented the same deep learning neural network that was used in DarkCovidNet [20]. Table 5 lists the parameters used to design and develop DarkCovidNet. The penultimate row in Table 4 shows the classification performance achieved using DarkCovidNet [20]. As the last two rows in the table indicate, the proposed model outperformed DarkCovidNet [20] by $1.94 \%$. This performance improvement serves as empirical proof that the proposed model provides a better deep neural network structure compared to the structure of DarkCovidNet [20] for classifying COVID-19 cases. 


\section{Conclusion and future directions}

Monitoring and tracking patient health status is a critical target in the ongoing COVID-19 pandemic. The advent of modern and efficient computational intelligence techniques like deep learning neural networks and high-performance distributed computing facilities can aid in automatically tracking and detecting COVID19 cases. This study proposes a new model for accurately classify and diagnosing COVID-19 using state-of the art deep learning models, especially GAs and CNNs. The proposed CGForCovid model will help to reduce clinician workload significantly during the pandemic and will work as a decision support system. In the future, the model can be extended to help rehabilitate affected patients in a timely manner. In this regard, we plan to implement the proposed framework as a protocol and develop a Raspberry Pi-based device to be deployed at a number of hospitals.

Acknowledgements This work was supported by King Saud University, Riyadh, Saudi Arabia, through Researchers Supporting Project number RSP-2021/18 and MEIF-SCI FY2021 grant of University of Maine System (UMS), USA.

\section{Declarations}

Ethical approval It does not apply.

Conflicts of interest The authors declare that they have no conflict of interest.

Informed consent It does not apply.

\section{References}

1. Akada T, Fujimori K (2021) Designing microwave circuits using genetic algorithms accelerated by convolutional neural networks. In: 2020 50th European Microwave Conference (EuMC), IEEE, pp 61-64

2. Apostolopoulos ID, Mpesiana TA (2020) Covid-19: automatic detection from x-ray images utilizing transfer learning with convolutional neural networks. Phys Eng Sci Med 43(2):635-640

3. Bakhshi A, Noman N, Chen Z, Zamani M, Chalup S (2019) Fast automatic optimisation of CNN architectures for image classification using genetic algorithm. In: 2019 IEEE congress on evolutionary computation (CEC), IEEE, pp 1283-1290

4. Blanco R, Malagón P, Cilla JJ, Moya JM (2018) Multiclass network attack classifier using cnn tuned with genetic algorithms. 2018 28th International symposium on power and timing modeling. Optimization and Simulation (PATMOS), IEEE, pp 177-182

5. Cabada RZ, Rangel HR, Estrada MLB, Lopez HMC (2020) Hyperparameter optimization in CNN for learning-centered emotion recognition for intelligent tutoring systems. Soft Comput 24(10):7593-7602

6. Chatterjee R, Maitra T, Islam SH, Hassan MM, Alamri A, Fortino G (2019) A novel machine learning based feature selection for motor imagery eeg signal classification in internet of medical things environment. Future Gener Comput Syst 98:419-434

7. Article published on News18 (2020) Know the result time of COVID-19 rt-pcr test and why it takes long. https://www.news18.com/news/india/know-the-result-time-of-covid-19-rt-pcr-test-and-why-ittakes-long-3142547.html 
8. Falaschi Z, Danna PS, Arioli R, Pasché A, Zagaria D, Percivale I, Tricca S, Barini M, Aquilini F, Andreoni S et al (2020) Chest CT accuracy in diagnosing COVID-19 during the peak of the Italian epidemic: A retrospective correlation with RT-PCR testing and analysis of discordant cases. European journal of radiology 130(109):192

9. Fang Y, Zhang H, Xie J, Lin M, Ying L, Pang P, Ji W (2020) Sensitivity of chest CT for COVID-19: comparison to RT-PCR. Radiology 296(2):E115-E117

10. Fawcett T (2006) An introduction to ROC analysis. Pattern Recognit Letters 27(8):861-874

11. Hassan MR, Hassan MM, Altaf M, Yeasar MS, Hossain MI, Fatema K, Shaharin R, Ahmed AF (2021) B5g-enabled distributed artificial intelligence on edges for COVID-19 pandemic outbreak prediction. IEEE Netw 35(3):48-55

12. Hemdan EED, Shouman MA, Karar ME (2020) Covidx-net: A framework of deep learning classifiers to diagnose COVID-19 in X-ray images. arXiv preprint arXiv:200311055

13. Huang C, Wang Y, Li X, Ren L, Zhao J, Hu Y, Zhang L, Fan G, Xu J, Gu X et al (2020) Clinical features of patients infected with 2019 novel coronavirus in Wuhan, china. The lancet 395(10223):497-506

14. Jaderberg M, Dalibard V, Osindero S, Czarnecki WM, Donahue J, Razavi A, Vinyals O, Green T, Dunning I, Simonyan K, et al. (2017) Population based training of neural networks. arXiv preprint arXiv: 171109846

15. Joseph Paul Cohen LD Paul Morrison (2020) Covid-19 image data collection. arXiv preprint arXiv: 200311597

16. Lei S, Hu H, Chen B, Lin Z, Tian J, Yang W, Tang P, Qiu X (2021) Analytical scannable-shaped beam pattern synthesis via superposition principle. IET Microw, Antennas Propag 15(6):600-605

17. Loussaief S, Abdelkrim A (2018) Convolutional neural network hyper-parameters optimization based on genetic algorithms. Int J Adv Comput Sci Appl 9(10):252-266

18. Narin A, Kaya C, Pamuk Z (2020) Automatic detection of coronavirus disease (covid-19) using X-ray images and deep convolutional neural networks. arXiv preprint arXiv:200310849

19. Ng MY, Lee EY, Yang J, Yang F, Li X, Wang H, Lui MMs, Lo CSY (2020) Imaging profile of the COVID-19 infection: radiologic findings and literature review. Radiol Cardiothorac Imagin 2(1):e200034

20. Ozturk T, Talo M, Yildirim EA, Baloglu UB, Yildirim O, Acharya UR (2020) Automated detection of COVID-19 cases using deep neural networks with X-ray images. Comput Biol Med 121(103):792

21. Panwar H, Gupta P, Siddiqui MK, Morales-Menendez R, Singh V (2020) Application of deep learning for fast detection of COVID-19 in X-rays using nCOVnet. Chaos, Solitons Fractals 138(109):944

22. Ranney ML, Griffeth V, Jha AK (2020) Critical supply shortages-the need for ventilators and personal protective equipment during the COVID-19 pandemic. New Engl J Med 382(18):e41

23. Sajja PS (2021) Examples and applications on genetic algorithms. In: Illustrated Computational Intelligence, Springer, pp 155-189

24. Sethy PK, Behera SK, Ratha PK, Biswas P (2020) Detection of coronavirus disease (COVID-19) based on deep features and support vector machine. Int J Math, Eng Manag Sci

25. Singhal T (2020) A review of coronavirus disease-2019 (COVID-19). The Indian J Pediatr 87(4):281-286

26. Song Y, Zheng S, Li L, Zhang X, Zhang X, Huang Z, Chen J, Wang R, Zhao H, Zha Y et al (2021) Deep learning enables accurate diagnosis of novel coronavirus (COVID-19) with ct images. IEEE/ ACM Trans Comput Biol Bioinform

27. Sun Y, Xue B, Zhang M, Yen GG, Lv J (2020) Automatically designing CNN architectures using the genetic algorithm for image classification. IEEE Trans Cybern 50(9):3840-3854

28. Toraman S, Alakus TB, Turkoglu I (2020) Convolutional capsnet: A novel artificial neural network approach to detect covid-19 disease from x-ray images using capsule networks. Chaos, Solitons Fractals 140(110):122

29. Waheed A, Goyal M, Gupta D, Khanna A, Al-Turjman F, Pinheiro PR (2020) Covidgan: data augmentation using auxiliary classifier gan for improved COVID-19 detection. IEEE Access 8:91916-91923

30. Wang L, Lin ZQ, Wong A (2020) Covid-net: A tailored deep convolutional neural network design for detection of COVID-19 cases from chest X-ray images. Sci Rep 10(1):1-12 
31. Wang S, Kang B, Ma J, Zeng X, Xiao M, Guo J, Cai M, Yang J, Li Y, Meng X, et al. (2021) A deep learning algorithm using CT images to screen for corona virus disease (COVID-19). Euro Radiol pp 1-9

32. Wang Z, Sobey A (2020) A comparative review between genetic algorithm use in composite optimisation and the state-of-the-art in evolutionary computation. Composite Struct 233(111):739

33. Wu F, Zhao S, Yu B, Chen YM, Wang W, Song ZG, Hu Y, Tao ZW, Tian JH, Pei YY et al (2020) A new coronavirus associated with human respiratory disease in china. Nature 579(7798):265-269

34. Wu JMT, Tsai MH, Huang YZ, Islam SH, Hassan MM, Alelaiwi A, Fortino G (2019) Applying an ensemble convolutional neural network with savitzky-golay filter to construct a phonocardiogram prediction model. Appl Soft Comput 78:29-40

35. Xiao X, Yan M, Basodi S, Ji C, Pan Y (2020) Efficient hyperparameter optimization in deep learning using a variable length genetic algorithm. arXiv preprint arXiv:200612703

36. Xiao X, Huang H, Wang W (2021) Underwater wireless sensor networks: An energy-efficient clustering routing protocol based on data fusion and genetic algorithms. Appl Sci 11(1):312

37. Xie L, Yuille A (2017) Genetic CNN. In: Proceedings of 2017 IEEE International Conference on Computer Vision, Venice, Italy, IEEE, pp 1388-1397

38. Xu X, Jiang X, Ma C, Du P, Li X, Lv S, Yu L, Ni Q, Chen Y, Su J et al (2020) A deep learning system to screen novel coronavirus disease 2019 pneumonia. Engineering 6(10):1122-1129

39. Zheng C, Deng X, Fu Q, Zhou Q, Feng J, Ma H, Liu W, Wang X (2020) Deep learning-based detection for COVID-19 from chest CT using weak label. MedRxiv

Publisher's Note Springer Nature remains neutral with regard to jurisdictional claims in published maps and institutional affiliations.

\section{Authors and Affiliations}

\section{Md Rafiul Hassan ${ }^{1}$ - Walaa N. Ismail ${ }^{2}$. Ahmad Chowdhury ${ }^{3}$. Sharara Hossain ${ }^{4}$. Shamsul Huda ${ }^{5}$. Mohammad Mehedi Hassan ${ }^{6}$ (D)}

Mohammad Mehedi Hassan

mmhassan@ksu.edu.sa

Md Rafiul Hassan

md.hassan@maine.edu

Walaa N. Ismail

wlaanagy@gmail.com

Ahmad Chowdhury

ahmadchowdhury@iut-dhaka.edu

Sharara Hossain

shararhossain@iut-dhaka.edu

Shamsul Huda

shamsul.huda@deakin.edu.au

1 College of Arts and Sciences, University of Maine at Presque Isle, Presque Isle ME04769, USA

2 Faculty of Computers and Information, Minia University, Minia 61519, Egypt

3 Imagine Consulting Services LLC, Dayton, NJ, USA

4 Simply Retrofits, Ontario, Canada

5 School of Information Technology, Deakin University, Melbourne, Australia

6 Department of Information Systems, College of Computer and Information Sciences, King Saud University, Riyadh 11543, Saudi Arabia 